Article

\title{
Oscillation Theorems for Advanced Differential Equations with $p$-Laplacian Like Operators
}

\author{
Omar Bazighifan $1,2, *,+$ (iD) and Poom Kumam $3,4, *,+$ (iD \\ 1 Department of Mathematics, Faculty of Science, Hadhramout University, Hadhramout 50512, Yemen \\ 2 Department of Mathematics, Faculty of Education, Seiyun University, Hadhramout 50512, Yemen \\ 3 Center of Excellence in Theoretical and Computational Science (TaCS-CoE) and Department of Mathematics, \\ Faculty of Science, King Mongkuts University of Technology Thonburi (KMUTT), 126 Pracha Uthit Rd., \\ Bang Mod, Thung Khru, Bangkok 10140, Thailand \\ 4 Department of Medical Research, China Medical University Hospital, China Medical University, \\ Taichung 40402, Taiwan \\ * Correspondence: o.bazighifan@gmail.com (O.B.); poom.kumam@mail.kmutt.ac.th (P.K.) \\ + These authors contributed equally to this work.
}

Received: 7 April 2020; Accepted: 29 April 2020; Published: 19 May 2020

check for updates

\begin{abstract}
The main objective of this paper is to establish new oscillation results of solutions to a class of even-order advanced differential equations with a $p$-Laplacian like operator. The key idea of our approach is to use the Riccati transformation and the theory of comparison with first and second-order delay equations. Some examples are provided to illustrate the main results.
\end{abstract}

Keywords: oscillation; even-order; advanced differential equations; $p$-Laplacian equations

\section{Introduction}

We provide oscillation properties of even order advanced differential equation with a $p$-Laplacian like operator

$$
\left(a(v)\left|y^{(\kappa-1)}(v)\right|^{p-2} y^{(\kappa-1)}(v)\right)^{\prime}+\sum_{i=1}^{j} q_{i}(v) g\left(y\left(\eta_{i}(v)\right)\right)=0, \quad v \geq v_{0}
$$

where $j \geq 1, \kappa$ is even and $p>1$ is a real number. Throughout the paper, we assume that (1) satisfy the following assumptions.

L1: $\quad a \in C^{1}\left(\left[v_{0}, \infty\right), \mathbb{R}\right), a(v)>0, a^{\prime}(v) \geq 0$,

L2: $\quad q_{i}, \eta_{i} \in C\left(\left[v_{0}, \infty\right), \mathbb{R}\right), q_{i}(v) \geq 0, \eta_{i}(v) \geq v, \lim _{v \rightarrow \infty} \eta_{i}(v)=\infty, i=1,2, . ., j$,

L3: $\quad g \in C(\mathbb{R}, \mathbb{R})$ such that $g(x) /|x|^{p-2} x \geq k>0$, for $x \neq 0, k$ is a constant, and under the condition

$$
\int_{v_{0}}^{\infty} \frac{1}{a^{1 /(p-1)}(s)} \mathrm{d} s=\infty
$$

Definition 1. The function $y \in C^{\mathcal{K}-1}\left[v_{y}, \infty\right), v_{y} \geq v_{0}$, is called a solution of (1), if $\left|y^{(\kappa-1)}(v)\right|^{p-2} y^{(\kappa-1)}(v) \in C^{1}\left[v_{y}, \infty\right)$, for $a \in C^{1}\left(\left[v_{0}, \infty\right), \mathbb{R}\right), a(v)>0$ and $y(v)$ satisfies $(1)$ on $\left[v_{y}, \infty\right)$.

The $p$-Laplace equations have some applications in continuum mechanics, see [1-3]. Advanced differential equations naturally appear in models concerning physical, biological, and chemical phenomena, mechanical, see [4], and in the mathematical modeling of engineering problems, such as electrical power systems, see [5], materials, see [6], and energy, see [7]. 
During this decade, Several works have been accomplished in the development of the oscillation theory of higher order advanced equations by using the Riccati transformation and the theory of comparison between first and second-order delay equations, see [8-19]. Further, the oscillation theory of fourth and second order equations has been studied and developed by using integral averaging technique and the Riccati transformation, see [20-27].

Our aim of this paper is complement and improve the results contained in [28-30]. For this purpose we discuss first these results.

Moaaz et al. [31] considered the fourth-order differential equation

$$
\left(a(v)\left(y^{\prime \prime \prime}(v)\right)^{\beta}\right)^{\prime}+q(v) y^{\alpha}(\eta(v))=0,
$$

where $\beta, \alpha$ are quotients of odd positive integers.

Grace et al. [32] considered the equation

$$
\left(a(v)\left(y^{\prime \prime}(v)\right)^{\beta}\right)^{\prime \prime}+q(v) g(y(\eta(v)))=0,
$$

where $\eta(v) \leq v, \beta$ is a quotient of odd positive integers.

Zhang et al. in [3] studied the qualitative behavior of the fourth-order differential equation

$$
\left(a(v)\left(w^{\prime \prime \prime}(v)\right)^{\beta}\right)^{\prime}+q(v) w(\eta(v))=0,
$$

where $\eta(v) \leq v, \beta$ is a quotient of odd positive integers and they used the Riccati transformation.

Agarwal and Grace [28] considered the equation

$$
\left(\left(y^{(\kappa-1)}(v)\right)^{\beta}\right)^{\prime}+q(v) y^{\beta}(\eta(v))=0,
$$

where $\kappa$ is an even and they established some new oscillation criteria by using the comparison technique. Among others, they proved it oscillatory if

$$
\liminf _{v \rightarrow \infty} \int_{v}^{\eta(v)}(\eta(s)-s)^{\kappa-2}\left(\int_{\eta(v)}^{\infty} q(v) d v\right)^{1 / \beta} d s>\frac{(\kappa-2) !}{e} .
$$

Agarwal et al. in [29] extended the Riccati transformation to obtain new oscillatory criteria for ODE (4) under the condition

$$
\limsup _{v \rightarrow \infty} v^{\beta(\kappa-1)} \int_{v}^{\infty} q(s) d s>((\kappa-1) !)^{\beta} .
$$

The authors in [30] studied oscillatory behavior of (4) where $\beta=1$ and if there exists a function $\tau \in C^{1}\left(\left[v_{0}, \infty\right),(0, \infty)\right)$, also, they proved it oscillatory by using the Riccati transformation if

$$
\int_{v}^{\infty}\left(\tau(s) q(s)-\frac{(\kappa-2) !\left(\tau^{\prime}(s)\right)^{2}}{2^{3-2 \kappa} s^{\mathcal{K}-2} \tau(s)}\right) d s=\infty .
$$

The main aim of this paper is to establish new oscillation results of solutions to a class of even-order differential equations and they essentially complement and improve the results contained in [28-30].

The rest of the paper is organized as follows. In Section 2, three lemmas are given to prove the main results. In Section 3, we establish new oscillation results for (1), comparisons are carried out with oscillations of first and second-order delay differential equations and two examples are presented to illustrate the main results. Some conclusions are discussed in the last Section 4. 


\section{Some Auxiliary Lemmas}

We shall employ the following lemmas which are found in the references $[8,15,20,27]$ respectively:

Lemma 1. If $y^{(i)}(v)>0, i=0,1, \ldots, \kappa$, and $y^{(\kappa+1)}(v)<0$, then

$$
\frac{y(v)}{v^{\kappa} / \kappa !} \geq \frac{y^{\prime}(v)}{v^{\kappa-1} /(\kappa-1) !}
$$

Lemma 2. Suppose that $y \in C^{\kappa}\left(\left[v_{0}, \infty\right),(0, \infty)\right), y^{(\kappa)}$ is of a fixed sign on $\left[v_{0}, \infty\right), y^{(\kappa)}$ not identically zero and there exists $a v_{1} \geq v_{0}$ such that

$$
y^{(\kappa-1)}(v) y^{(\kappa)}(v) \leq 0
$$

for all $v \geq v_{1}$. If we have $\lim _{v \rightarrow \infty} y(v) \neq 0$, then there exists $v_{\theta} \geq v_{1}$ such that

$$
y(v) \geq \frac{\theta}{(\kappa-1) !} v^{\kappa-1}\left|y^{(\kappa-1)}(v)\right|,
$$

for every $\theta \in(0,1)$ and $v \geq v_{\theta}$.

Lemma 3. Suppose that $y$ is an eventually positive solution of (1). Then, there exist two possible cases:

$$
\begin{array}{ll}
\left(\mathbf{S}_{1}\right) & y(v)>0, y^{\prime}(v)>0, y^{\prime \prime}(v)>0, y^{(\kappa-1)}(v)>0, y^{(\kappa)}(v)<0, \\
\left(\mathbf{S}_{2}\right) & y(v)>0, y^{(r)}(v)>0, y^{(r+1)}(v)<0 \text { for all odd integer } \\
& r \in\{1,3, \ldots, \kappa-3\}, y^{(\kappa-1)}(v)>0, y^{(\kappa)}(v)<0,
\end{array}
$$

for $v \geq v_{1}$, where $v_{1} \geq v_{0}$ is sufficiently large.

Remark 1. Let the differential equation

$$
\left[a(v)\left(y^{\prime}(v)\right)^{\beta}\right]^{\prime}+q(v) y^{\beta}(g(v))=0, \quad v \geq v_{0}
$$

where $\beta>0$ is the ratio of odd positive integers, $a, q \in C\left(\left[v_{0}, \infty\right), \mathbb{R}^{+}\right)$, is nonoscillatory if and only if there exist a number $v \geq v_{0}$, and a function $\varsigma \in C^{1}([v, \infty), \mathbb{R})$, satisfying the inequality

$$
\varsigma^{\prime}(v)+\gamma a^{-1 / \beta}(v)(\varsigma(v))^{(1+\beta) / \beta}+q(v) \leq 0, \quad \text { on }[v, \infty) .
$$

\section{Comparison Theorems with Second/First-Order Equations}

Theorem 1. Assume that (2) holds. If the differential equations

$$
\left(\frac{(\kappa-2) ! a^{\frac{1}{(p-1)}}(v)}{\left(\theta v^{\kappa-2}\right)^{p-1}}\left(y^{\prime}(v)\right)^{p-1}\right)^{\prime}+k \sum_{i=1}^{j} q_{i}(v) y^{p-1}(v)=0
$$

for every $\theta \in(0,1)$, and

$$
y^{\prime \prime}(v)+y(v) \frac{1}{(\kappa-4) !} \int_{v}^{\infty}(\varsigma-v)^{\kappa-4}\left(\frac{1}{a(\varsigma)} \int_{\varsigma}^{\infty} \sum_{i=1}^{j} q_{i}(s) \mathrm{d} s\right)^{1 /(p-1)} \mathrm{d} \varsigma=0
$$

are oscillatory. Then, every solution of (1) is oscillatory.

Proof. On the contrary, assume that $y$ is a positive solution of (1). Then, we can suppose that $y(v)$ and $y\left(\eta_{i}(v)\right)$ are positive for all $v \geq v_{1}$ sufficiently large. From Lemma 3, we have two possible cases $\left(\mathbf{S}_{1}\right)$ and $\left(\mathbf{S}_{2}\right)$. 
Let case $\left(\mathbf{S}_{1}\right)$ holds. Using Lemma 2, we find

$$
y^{\prime}(v) \geq \frac{\theta}{2} v^{\kappa-2} y^{(\kappa-1)}(v),
$$

for every $\theta \in(0,1)$ and for all large $v$.

Define

$$
\varphi(v):=\tau(v)\left(\frac{a(v)\left(y^{(\kappa-1)}(v)\right)^{p-1}}{y^{p-1}(v)}\right),
$$

we see that $\varphi(v)>0$ for $v \geq v_{1}$, where $\tau \in C^{1}\left(\left[v_{0}, \infty\right),(0, \infty)\right)$ and

$$
\begin{aligned}
\varphi^{\prime}(v)= & \tau^{\prime}(v) \frac{a(v)\left(y^{(\kappa-1)}(v)\right)^{p-1}}{y^{p-1}(v)}+\tau(v) \frac{\left(a\left(y^{(\kappa-1)}\right)^{p-1}\right)^{\prime}(v)}{y^{p-1}(v)} \\
& -\beta \tau(v) \frac{y^{p-2}(v) y^{\prime}(v) a(v)\left(y^{(\kappa-1)}(v)\right)^{p-1}}{y^{2(p-1)}(v)} .
\end{aligned}
$$

Using (11) and (12), we obtain

$$
\begin{aligned}
\varphi^{\prime}(v) \leq & \frac{\tau_{+}^{\prime}(v)}{\tau(v)} \varphi(v)+\tau(v) \frac{\left(a(v)\left(y^{(\kappa-1)}(v)\right)^{p-1}\right)^{\prime}}{y^{p-1}(v)} \\
& -(p-1) \tau(v) \frac{\theta}{(\kappa-2) !} v^{\kappa-2} \frac{a(v)\left(y^{(\kappa-1)}(v)\right)^{p}}{y^{p}(v)} \\
\leq & \frac{\tau^{\prime}(v)}{\tau(v)} \varphi(v)+\tau(v) \frac{\left(a(v)\left(y^{(\kappa-1)}(v)\right)^{p-1}\right)^{\prime}}{y^{p-1}(v)} \\
& -\frac{(p-1) \theta v^{\kappa-2}}{(\kappa-2) !(\tau(v) a(v))^{\frac{1}{p-1}}} \varphi(v)^{\frac{p}{p-1}} .
\end{aligned}
$$

From (1) and (13), we obtain

$$
\varphi^{\prime}(v) \leq \frac{\tau^{\prime}(v)}{\tau(v)} \varphi(v)-k \tau(v) \frac{\sum_{i=1}^{j} q_{i}(v) y^{p-1}\left(\eta_{i}(v)\right)}{y^{p-1}(v)}-\frac{(p-1) \theta v^{\kappa-2}}{(\kappa-2) !(\tau(v) a(v))^{\frac{1}{p-1}}} \varphi(v)^{\frac{p}{p-1}} .
$$

Note that $y^{\prime}(v)>0$ and $\eta_{i}(v) \geq v$, thus, we find

$$
\varphi^{\prime}(v) \leq \frac{\tau^{\prime}(v)}{\tau(v)} \varphi(v)-k \tau(v) \sum_{i=1}^{j} q_{i}(v)-\frac{(p-1) \theta v^{\kappa-2}}{(\kappa-2) !(\tau(v) a(v))^{\frac{1}{p-1}}} \varphi(v)^{\frac{p}{p-1}} .
$$

If we set $\tau(v)=k=1$ in (14), then we find

$$
\varphi^{\prime}(v)+\frac{(p-1) \theta v^{\kappa-2}}{(\kappa-2) !(\tau(v) a(v))^{\frac{1}{p-1}}} \varphi(v)^{\frac{p}{p-1}}+\sum_{i=1}^{j} q_{i}(v) \leq 0 .
$$

Therefore, we note that the Equation (9) is nonoscillatory, which is a contradiction.

Let case $\left(\mathbf{S}_{2}\right)$ holds. Define

$$
\psi(v):=\vartheta(v) \frac{y^{\prime}(v)}{y(v)},
$$


we see that $\psi(v)>0$ for $v \geq v_{1}$, where $\vartheta \in C^{1}\left(\left[v_{0}, \infty\right),(0, \infty)\right)$. By differentiating of $\psi(v)$, we find

$$
\psi^{\prime}(v)=\frac{\vartheta^{\prime}(v)}{\vartheta(v)} \psi(v)+\vartheta(v) \frac{y^{\prime \prime}(v)}{y(v)}-\frac{1}{\vartheta(v)} \psi(v)^{2} .
$$

Now, we integrating (1) from $v$ to $m$ and using $y^{\prime}(v)>0$, we find

$$
a(m)\left(y^{(\kappa-1)}(m)\right)^{p-1}-a(v)\left(y^{(\kappa-1)}(v)\right)^{p-1}=-\int_{v}^{m} \sum_{i=1}^{j} q_{i}(s) g\left(y\left(\eta_{i}(s)\right)\right) d s .
$$

By virtue of $y^{\prime}(v)>0$ and $\eta_{i}(v) \geq v$, we get

$$
a(m)\left(y^{(\kappa-1)}(m)\right)^{p-1}-a(v)\left(y^{(\kappa-1)}(v)\right)^{p-1} \leq-k y^{p-1}(v) \int_{v}^{u} \sum_{i=1}^{j} q_{i}(s) d s .
$$

Letting $m \rightarrow \infty$, we see that

$$
a(v)\left(y^{(\kappa-1)}(v)\right)^{p-1} \geq k y^{p-1}(v) \int_{v}^{\infty} \sum_{i=1}^{j} q_{i}(s) \mathrm{d} s
$$

and so

$$
y^{(\kappa-1)}(v) \geq y(v)\left(\frac{k}{a(v)} \int_{v}^{\infty} \sum_{i=1}^{j} q_{i}(s) \mathrm{d} s\right)^{1 /(p-1)} .
$$

Integrating again from $v$ to $\infty$ for a total of $(\kappa-4)$ times, we get

$$
y^{\prime \prime}(v)+\frac{y(v)}{(\kappa-4) !} \int_{v}^{\infty}(\varsigma-v)^{\kappa-4}\left(\frac{k}{a(\varsigma)} \int_{\varsigma}^{\infty} \sum_{i=1}^{j} q_{i}(s) \mathrm{d} s\right)^{1 /(p-1)} \mathrm{d} \varsigma \leq 0 .
$$

From (15) and (16), we obtain

$$
\psi^{\prime}(v) \leq \frac{\vartheta^{\prime}(v)}{\vartheta(v)} \psi(v)-\frac{\vartheta(v)}{(\kappa-4) !} \int_{v}^{\infty}(\varsigma-v)^{\kappa-4}\left(\frac{k}{a(\varsigma)} \int_{\varsigma}^{\infty} \sum_{i=1}^{j} q_{i}(s) \mathrm{d} s\right)^{1 /(p-1)} \mathrm{d} \varsigma-\frac{1}{\vartheta(v)} \psi(v)^{2} .
$$

If we now set $\vartheta(v)=k=1$ in (17), then we obtain

$$
\psi^{\prime}(v)+\psi^{2}(v)+\frac{1}{(\kappa-4) !} \int_{v}^{\infty}(\varsigma-v)^{\kappa-4}\left(\frac{1}{a(\varsigma)} \int_{\varsigma}^{\infty} \sum_{i=1}^{j} q_{i}(s) \mathrm{d} s\right)^{1 /(p-1)} \mathrm{d} \varsigma \leq 0 .
$$

Hence, we see that the Equation (10) is nonoscillatory, which is a contradiction. Theorem 1 is proved.

Remark 2. It is well known (see [23]) that if

$$
\int_{v_{0}}^{\infty} \frac{1}{a(v)} \mathrm{d} v=\infty, \text { and } \liminf _{v \rightarrow \infty}\left(\int_{v_{0}}^{v} \frac{1}{a(s)} \mathrm{d} s\right) \int_{v}^{\infty} q(s) \mathrm{d} s>\frac{1}{4},
$$

then Equation (8) with $p=2$ is oscillatory.

Based on the above results and Theorem 1, we can easily obtain the following Hille and Nehari type oscillation criteria for (1) with $\beta=1$. 
Theorem 2. Let $p=2, k=1$. Assume that (2) holds. If

$$
\int_{v_{0}}^{\infty} \frac{\theta v^{\kappa-2}}{(\kappa-2) ! a(v)} \mathrm{d} v=\infty
$$

and

$$
\liminf _{v \rightarrow \infty}\left(\int_{v_{0}}^{v} \frac{\theta s^{\kappa-2}}{(\kappa-2) ! a(s)} \mathrm{d} s\right) \int_{v}^{\infty} \sum_{i=1}^{j} q_{i}(s) \mathrm{d} s>\frac{1}{4}
$$

also, if

$$
\liminf _{v \rightarrow \infty} \int_{v_{0}}^{v} \frac{1}{(\kappa-4) !} \int_{v}^{\infty}(\varsigma-v)^{\kappa-4}\left(\frac{1}{a(\varsigma)} \int_{\varsigma}^{\infty} \sum_{i=1}^{j} q_{i}(s) \mathrm{d} s\right) \mathrm{d} \varsigma \mathrm{d} v>\frac{1}{4},
$$

for all constant $\theta \in(0,1)$. Then all solutions of (1) are oscillatory.

In the theorem, we compare the oscillatory behavior of (1) with the first-order differential equations:

Theorem 3. Assume that (2) holds. If the differential equations

$$
x^{\prime}(v)+k \sum_{i=1}^{j} q_{i}(v)\left(\frac{\theta v^{\kappa-2}}{(\kappa-2) ! a^{1 / \beta}(v)}\right)^{p-1} x(\eta(v))=0
$$

and

$$
z^{\prime}(v)+z(v) \frac{v}{(\kappa-4) !} \int_{v}^{\infty}(\varsigma-v)^{\kappa-4}\left(\frac{k}{a(\varsigma)} \int_{\varsigma}^{\infty} \sum_{i=1}^{j} q_{i}(s) \mathrm{d} s\right)^{1 /(p-1)} \mathrm{d} \varsigma=0
$$

are oscillatory for all constant $\theta \in(0,1)$. Then all solutions of $(1)$ are oscillatory.

Proof. On the contrary, assume that $y$ is a positive solution of (1). Then, we can suppose that $y(v)$ and $y\left(\eta_{i}(v)\right)$ are positive for all $v \geq v_{1}$ sufficiently large. From Lemma 3, we have two possible cases $\left(\mathbf{S}_{1}\right)$ and $\left(\mathbf{S}_{2}\right)$.

In the case where $\left(\mathbf{S}_{1}\right)$ holds, from Lemma 2, we see

$$
y(v) \geq \frac{\theta v^{\kappa-2}}{(\kappa-2) ! a^{1 /(p-1)}(v)}\left(a^{1 /(p-1)}(v) y^{(\kappa-1)}(v)\right),
$$

for every $\theta \in(0,1)$ and for all large $v$. Thus, if we set

$$
x(v)=a(v)\left(y^{(\kappa-1)}(v)\right)^{p-1}>0
$$

then we see that $\psi$ is a positive solution of the inequality

$$
x^{\prime}(v)+k \sum_{i=1}^{j} q_{i}(v)\left(\frac{\theta v^{\kappa-2}}{(\kappa-2) ! a^{1 /(p-1)}(v)}\right)^{p-1} x(\eta(v)) \leq 0 .
$$

From [24] (Theorem 1), we see that the Equation (20) also has a positive solution and it is a contradiction.

In the case where $\left(\mathbf{S}_{2}\right)$ holds. From Lemma 1, we get

$$
y(v) \geq v y^{\prime}(v)
$$


From (16) and (23), we get

$$
y^{\prime \prime}(v)+y^{\prime}(v) \frac{v}{(\kappa-4) !} \int_{v}^{\infty}(\varsigma-v)^{\kappa-4}\left(\frac{k}{a(\varsigma)} \int_{\varsigma}^{\infty} \sum_{i=1}^{j} q_{i}(s) \mathrm{d} s\right)^{1 /(p-1)} \mathrm{d} \varsigma \leq 0 .
$$

Now, we set

$$
z(v)=y^{\prime}(v) .
$$

Thus, we find $\psi$ is a positive solution of the inequality

$$
z^{\prime}(v)+z(v) \frac{v}{(\kappa-4) !} \int_{v}^{\infty}(\varsigma-v)^{\kappa-4}\left(\frac{k}{a(\zeta)} \int_{\zeta}^{\infty} \sum_{i=1}^{j} q_{i}(s) \mathrm{d} s\right)^{1 /(p-1)} \mathrm{d} \varsigma \leq 0
$$

It is well known (see [24] (Theorem 1)) that the Equation (21) also has a positive solution and it is a contradiction.

The proof is complete.

Corollary 1. Let (2) holds. If

$$
\liminf _{v \rightarrow \infty} \int_{\eta_{i}(v)}^{v} \sum_{i=1}^{j} q_{i}(s)\left(\frac{\theta v^{\kappa-2}}{(\kappa-2) ! a^{1 /(p-1)}(v)}\right)^{p-1} \mathrm{~d} s>\frac{((\kappa-1) !)^{p-1}}{\mathrm{e}}
$$

and

$$
\liminf _{v \rightarrow \infty} \int_{\eta_{i}(v)}^{v} \frac{s}{(\kappa-4) !} \int_{v}^{\infty}(\varsigma-v)^{\kappa-4}\left(\frac{k}{a(\varsigma)} \int_{\varsigma}^{\infty} \sum_{i=1}^{j} q_{i}(s) \mathrm{d} s\right)^{1 /(p-1)} \mathrm{d} \varsigma \mathrm{d} s>\frac{1}{\mathrm{e}^{\prime}}
$$

for all constant $\theta \in(0,1)$. Then all solutions of (1) are oscillatory.

Let us consider the differential equation

Example 1. Let the equation

$$
y^{(4)}(v)+\frac{q_{0}}{v^{4}} y(3 v)=0, v \geq 1
$$

where $q_{0}>0$ is a constant. Note that $p=2, \kappa=4, a(v)=1, q(v)=q_{0} / v^{4}$ and $\eta(v)=3 v$. If we set $k=1$, then condition (18) becomes

$$
\begin{aligned}
\liminf _{v \rightarrow \infty}\left(\int_{v_{0}}^{v} \frac{\theta s^{\kappa-2}}{(\kappa-2) ! a(s)} \mathrm{d} s\right) \int_{v}^{\infty} \sum_{i=1}^{j} q_{i}(s) \mathrm{d} s & =\liminf _{v \rightarrow \infty}\left(\frac{v^{3}}{3}\right) \int_{v}^{\infty} \frac{q_{0}}{s^{4}} d s \\
& =\frac{q_{0}}{9}>\frac{1}{4}
\end{aligned}
$$

and condition (19) becomes

$$
\begin{aligned}
\liminf _{v \rightarrow \infty} \int_{v_{0}}^{v} \frac{1}{(\kappa-4) !} \int_{v}^{\infty}(\varsigma-v)^{\kappa-4}\left(\frac{1}{a(\varsigma)} \int_{\varsigma}^{\infty} \sum_{i=1}^{j} q_{i}(s) \mathrm{d} s\right)^{1 /(p-1)} \mathrm{d} \varsigma \mathrm{d} v & =\liminf _{v \rightarrow \infty}\left(\frac{q_{0}}{6 v}\right) \\
& =\frac{q_{0}}{6}>\frac{1}{4}
\end{aligned}
$$

Therefore, from Theorem 2, all the solutions of the equation (27) is oscillatory if $q_{0}>2.25$. 
Remark 3. We compare our result with the known related criteria for oscillation of this equation as follows:

\begin{tabular}{|l|c|c|c|c|}
\hline The condition & $(5)$ & $(6)$ & $(7)$ & our condition \\
\hline The criterion & $q_{0}>13.6$ & $q_{0}>18$ & $q_{0}>576$ & $q_{0}>2.25$ \\
\hline
\end{tabular}

Therefore, our result improve the results contained in [28-30].

Example 2. Consider the differential equation

$$
y^{(4)}(v)+\frac{q_{0}}{v^{4}} y(2 v)=0, v \geq 1
$$

where $q_{0}>0$ is a constant. Note that $p=2, \kappa=4, a(v)=1, q(v)=q_{0} / v^{4}$ and $\eta(v)=2 v$. If we set $k=1$, then condition (18) becomes

$$
\frac{q_{0}}{9}>\frac{1}{4}
$$

Therefore, from Theorem 2, all solution equation (28) is oscillatory if $q_{0}>2.25$.

Remark 4. We compare our result with the known related criteria for oscillation of this equation as follows:

\begin{tabular}{|l|c|c|c|c|}
\hline The condition & $(5)$ & $(6)$ & $(7)$ & our condition \\
\hline The criterion & $q_{0}>25.5$ & $q_{0}>18$ & $q_{0}>1728$ & $q_{0}>2.25$ \\
\hline
\end{tabular}

Therefore, our result improve the results contained in [28-30].

\section{Conclusions}

In this article, we study the oscillatory behavior of a class of non-linear even-order differential equations with a $p$-Laplacian like operator and establish sufficient conditions for oscillation of a even-order differential equation by using the theory of comparison with first and second-order delay equations and Riccati substitution technique.

For researchers interested in this field, and as part of our future research, there is a nice open problem which is finding new results in the following case:

$$
\int_{v_{0}}^{\infty} \frac{1}{a^{1 /(p-1)}(s)} \mathrm{d} s<\infty .
$$

For all this there is some research in progress.

Author Contributions: O.B.: Writing original draft, and writing review and editing. P.K.: Formal analysis, writing review and editing, funding and supervision. All authors have read and agreed to the published version of the manuscript.

Funding: The authors received no direct funding for this work.

Acknowledgments: The authors acknowledge the financial support provided by the Center of Excellence in Theoretical and Computational Science (TaCS-CoE), KMUTT. The authors thank the reviewers for for their useful comments, which led to the improvement of the content of the paper.

Conflicts of Interest: There are no competing interests between the authors.

\section{References}

1. Aronsson, G.; Janfalk, U. On Hele-Shaw flow of power-law fluids. Eur. J. Appl. Math. 1992, 3, $343-366$. [CrossRef]

2. Li, T.; Baculikova, B.; Dzurina, J.; Zhang, C. Oscillation of fourth order neutral differential equations with p-Laplacian like operators. Bound. Value Probl. 2014, 56, 41-58. [CrossRef]

3. Zhang, C.; Agarwal, R.P.; Li, T. Oscillation and asymptotic behavior of higher-order delay differential equations with p -Laplacian like operators. J. Math. Anal. Appl. 2014, 409, 1093-1106. [CrossRef] 
4. Grace, S.; Dzurina, J.; Jadlovska, I.; Li, T. On the oscillation of fourth order delay differential equations. Adv. Differ. Equ. 2019, 118, 1-15. [CrossRef]

5. Tzounas, G.; Dassios, I.; Milano, F. Modal Participation Factors of Algebraic Variables. IEEE Trans. Power Syst. 2020, 35, 742-750. [CrossRef]

6. Dassios, I. Stability of Bounded Dynamical Networks with Symmetry. Symmetry 2018, 10, 121. [CrossRef]

7. Hale, J.K. Theory of Functional Differential Equations; Springer: New York, NY, USA, 1977.

8. Agarwal, R.; Shieh, S.L.; Yeh, C.C. Oscillation criteria for second order retarde ddifferential equations. Math. Comput. Model. 1997, 26,1-11. [CrossRef]

9. Bohner, M.; Grace, S.R.; Jadlovska, I. Oscillation criteria for second-order neutral delay differential equations. Electron. J. Qual. Theory Differ. Equ. 2017, 60,1-12. [CrossRef]

10. Sugie, J. A remark on Philos-type oscillation criteria for differential equations. Electron. J. Qual. Theory Differ. Equ. 2020, 18, 1-7. [CrossRef]

11. Bazighifan, O. An Approach for Studying Asymptotic Properties of Solutions of Neutral Differential Equations. Symmetry 2020, 12, 555. [CrossRef]

12. Baculikova, B.; Dzurina, J.; Graef, J.R. On the oscillation of higher-order delay differential equations. Math. Slovaca 2012, 187, 387-400. [CrossRef]

13. Bazighifan, O.; Dassios, I. Riccati Technique and Asymptotic Behavior of Fourth-Order Advanced Differential Equations. Mathematics 2020, 8, 590. [CrossRef]

14. Bazighifan, O.; Cesarano, C. A Philos-Type Oscillation Criteria for Fourth-Order Neutral Differential Equations. Symmetry 2020, 12, 379. [CrossRef]

15. Bazighifan, O.; Postolache, M. An improved conditions for oscillation of functional nonlinear differential equations. Mathematics 2020, 8, 552. [CrossRef]

16. Cesarano, C.; Pinelas, S.; Al-Showaikh, F.; Bazighifan, O. Asymptotic Properties of Solutions of Fourth-Order Delay Differential Equations. Symmetry 2019, 11, 628. [CrossRef]

17. Bazighifan, O.; Abdeljawad, T. Improved Approach for Studying Oscillatory Properties of Fourth-Order Advanced Differential Equations with p-Laplacian Like Operator. Mathematics 2020, 8, 656. [CrossRef]

18. Bazighifan, O.; Ruggieri, M.; Scapellato, A. An Improved Criterion for the Oscillation of Fourth-Order Differential Equations. Mathematics 2020, 8, 610. [CrossRef]

19. Gyori, I.; Ladas, G. Oscillation Theory of Delay Differential Equations with Applications; Clarendon Press: Oxford, UK, 1991.

20. Moaaz, O.; Awrejcewicz, J.; Bazighifan, O. A New Approach in the Study of Oscillation Criteria of Even-Order Neutral Differential Equations. Mathematics 2020, 12, 197. [CrossRef]

21. Moaaz, O.; Kumam, P.; Bazighifan, O. On the Oscillatory Behavior of a Class of Fourth-Order Nonlinear Differential Equation. Symmetry 2020, 12, 524. [CrossRef]

22. Moaaz, O.; Elabbasy, E.M.; Muhib, A. Oscillation criteria for even-order neutral differential equations with distributed deviating arguments. Adv. Differ. Equ. 2019, 2019, 297. [CrossRef]

23. Nehari, Z. Oscillation criteria for second order linear differential equations. Trans. Am. Math. Soc. 1957, 85, 428-445. [CrossRef]

24. Philos, C. On the existence of nonoscillatory solutions tending to zero at $\infty$ for differential equations with positive delay. Arch. Math. 1981, 36, 168-178. [CrossRef]

25. Rehak, P. How the constants in Hille-Nehari theorems depend on time scales. Adv. Differ. Equ. 2006, 2006, 1-15. [CrossRef]

26. Zhang, C.; Agarwal, R.P.; Bohner, M.; Li, T. New results for oscillatory behavior of even-order half-linear delay differential equations. Appl. Math. Lett. 2013, 26, 179-183. [CrossRef]

27. Zhang, C.; Li, T.; Sun, B.; Thandapani, E. On the oscillation of higher-order half-linear delay differential equations. Appl. Math. Lett. 2011, 24, 1618-1621. [CrossRef]

28. Agarwal, R.; Grace, S.R. Oscillation theorems for certain functional differential equations of higher order. Math. Comput. Model. 2004, 39, 1185-1194. [CrossRef]

29. Agarwal, R.; Grace, S.R.; O'Regan, D. Oscillation criteria for certain $\mathrm{n}$ th order differential equations with deviating arguments. J. Math. Anal. Appl. 2001, 262, 601-622. [CrossRef]

30. Grace, S.R.; Lalli, B.S. Oscillation theorems for nth-order differential equations with deviating arguments. Proc. Am. Math. Soc. 1984, 90, 65-70. 
31. Moaaz, O.; Dassios, I.; Bazighifan, O.; Muhib, A. Oscillation Theorems for Nonlinear Differential Equations of Fourth-Order. Mathematics 2020, 8, 520. [CrossRef]

32. Grace, S.R.; Bohner, M.; Liu, A. Oscillation criteria for fourth-order functional differential equations. Math. Slovaca 2013, 63, 1303-1320. [CrossRef]

(C) 2020 by the authors. Licensee MDPI, Basel, Switzerland. This article is an open access article distributed under the terms and conditions of the Creative Commons Attribution (CC BY) license (http:/ / creativecommons.org/licenses/by/4.0/). 